\title{
Bond Quality Inspection of Bonded Surfaces using Microwave NDE
}

\author{
Asha Gokul ${ }^{1 *}$, K. Srinivas ${ }^{2}$ and J. Dhanasekaran ${ }^{3}$ \\ 1,2,3 Scientists, Directorate of NDE \\ Dr. APJ Abdul Kalam Missile Complex, Advanced Systems Laboratory, DRDO, Hyderabad-500058, Telangana, India \\ *ashagokul@asl.drdo.in
}

\begin{abstract}
Solid rocket motor is an essential component of an aerospace structure. Bond quality of motor casing and insulation layer plays an important role in the mission performance. Different NDE techniques, such as ultrasonics, thermography, X-ray radiography are employed for evaluating the bond quality of the casing. Depending on the type of material, their characteristic features and accessibility of the test object, suitable technique is employed. Microwave non-destructive evaluation (MWNDE) is an emerging NDE technique for characterizing and inspecting dielectric structures. Microwave NDE finds application in the areas of dielectric material characterization, determining thickness variation, defect detection and bond quality inspection. Bond quality of fiber reinforced polymer (FRP) composite or metal with insulation layer inspected by near-field reflection microwave NDE technique is presented in this paper. Results of inspected structures by swept frequency reflection microwave NDE technique in the frequency range $8.2-12.4 \mathrm{GHz}$ (X-band) and $12.4-18 \mathrm{GHz}(\mathrm{Ku}-\mathrm{band})$ respectively are presented.
\end{abstract}

Keywords: Microwave NDE, near-field reflection method, bond quality inspection and swept frequency NDE technique

\section{Introduction}

The solid rocket motor is an essential component of an aerospace structure. Fiber-reinforced polymer (FRP) composite or metal forms the motor casing. The bond quality of the motor casing and insulation layer determines the aerospace mission objectives. For efficient service of the product for the intended purpose, the physical and functional evaluation must be done. Non-destructive evaluation (NDE) plays an important role in characterizing and confirming the quality of the product, for its functionality and applications. Ultrasonics, Thermography, X-ray radiography are the most common NDE techniques for evaluating bond quality. Depending on the type of material, their characteristic features and accessibility of the test object, a suitable technique is employed. Microwave Non-destructive evaluation (MWNDE) is an emerging NDE technique for characterizing and inspecting the dielectric structures [1]. Microwave NDE operates in the frequency range of $1 \mathrm{GHz}$ $40 \mathrm{GHz}$ of the electromagnetic spectrum. The following features of microwaves makes it attractive as an NDE tool:

- Ability to penetrate dielectric materials

- No couplant is required during testing

- Testing is done in either near-field or far-field of the probe

- Single-sided (Reflection method) or two- sided (Transmission reflection method) inspection

- Provides contact mode or non-contact mode of testing

- Cost-effective inspecting setup

Areas of application of microwave NDE include dielectric material characterization, monitoring or determining thickness variation, defect detection and bond quality inspection. Bond quality of fiber-reinforced polymer (FRP) composite or metal with insulation layer inspected by nearfield reflection microwave NDE technique is presented in this paper. Results of inspected structures by swept- frequency microwave NDE technique in the frequency range of $8.2-18 \mathrm{GHz}$ are presented.

\section{Theoretical background}

The dielectric material is defined as a non-conductor which stores charge from an external electric field. A dielectric material is characterized by dielectric properties namely, dielectric constant and loss tangent at a particular frequency. When microwaves are incident on a dielectric material, part of the energy is reflected, part is transmitted through the material and remaining is absorbed. The distribution of energy into these three categories is due to variation in the dielectric properties of the material from that of the external medium. The interaction of microwaves with the dielectric material in the form of transmission, reflection, and absorption is the basis for microwave NDE [2].

Near-field reflection microwave NDE is employed for bond quality inspection. Further, testing can be done in either contact or non-contact mode. Dielectric material/structure backed by conductor shown in Figure 1 is the testing configuration, where the reflection coefficient, $\mathrm{S}_{11}$ is the measurable parameter [1]. The reflection coefficient consists of magnitude and phase information as a function of operating/testing frequency. For material with low dielectric properties, material backed by conductor configuration is used to enhance the reflected signal. As the test configuration involves conductor backup, this leads to a single-sided inspection of the structure. Figure 2 shows the standard X-band $(8.2-12.4 \mathrm{GHz})$ Open-ended rectangular waveguide $(\mathrm{OEWG})$ probe operating in a dominant $\mathrm{TE}_{10}$ mode. For near-field measurement, a square flange with sides greater than one-wavelength in free space is a good approximation to the theoretical assumption of an infinite flange where fields exist within the radiating aperture [1]. To enhance the detection sensitivity, operating frequency incase of both contact and non-contact methods, and stand-off distance incase of the non-contact method is optimized. 


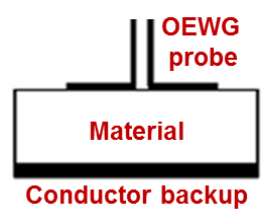

(a) Contact method

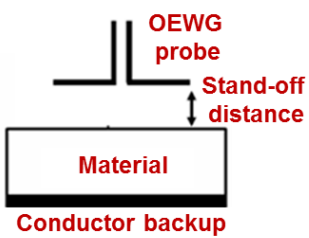

(b) Non-contact method
Fig. 1. Near-field reflection microwave NDE test configuration.

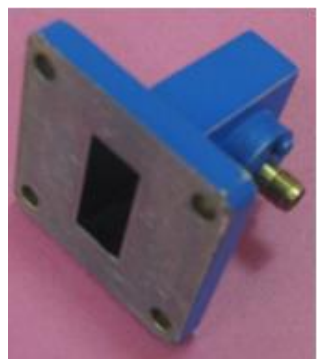

Fig. 2. X-band (8.2-12.4GHz) open-ended rectangular waveguide (OEWG) probe

\section{Experiment and results}

The experimental setup for Near-field reflection microwave NDE in contact and non-contact modes are shown in Figures 3 and 4, respectively. The setup consists of Keysight Field Fox microwave network analyzer (Portable MNA), coaxial cables, open-ended rectangular waveguide probe, waveguide calibration kit and sample under test backed by the conductor [3]. The experimental setup is calibrated for the test frequency and the reflection coefficient of the conductor is measured. The reflection coefficient of conductor consists of magnitude and phase, which is the reference.

Inspection of bond quality is carried out by measuring the reflection characteristics for each of the following structures: (a) E-glass epoxy composite-silicone rubber (b) 15CDV6 metal- rocasin rubber and (c) sub-scaled model of composite pressure vessel, in both contact and non-contact modes respectively. Figure 5 shows the configuration of the test sample with bond and debond regions respectively.

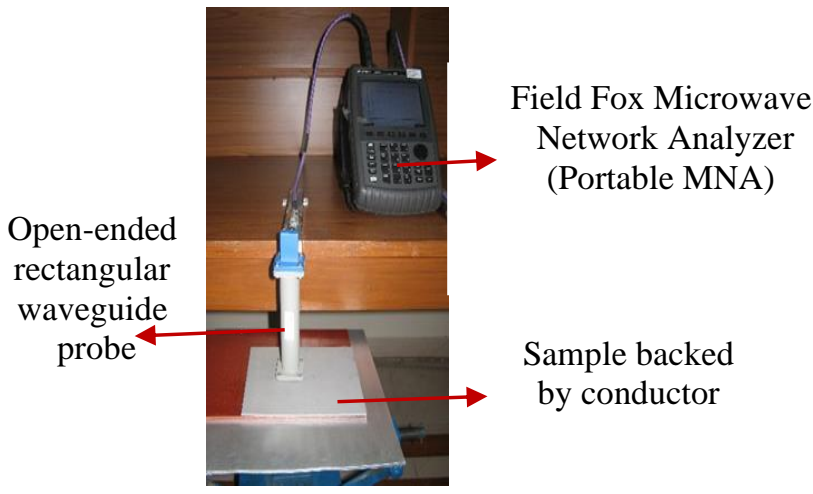

Fig. 3. Measured and simulated VSWR plots of dual band-notched UWB antenna

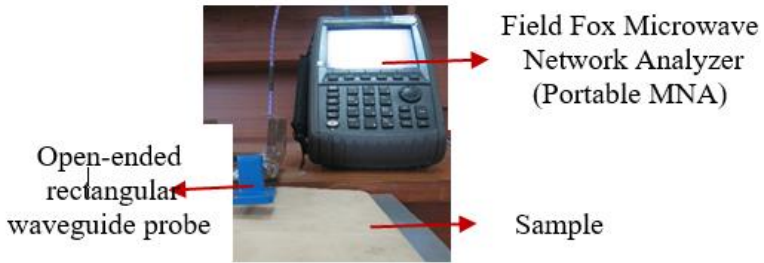

Fig. 4. Near-field reflection microwave NDE (non-contact mode)

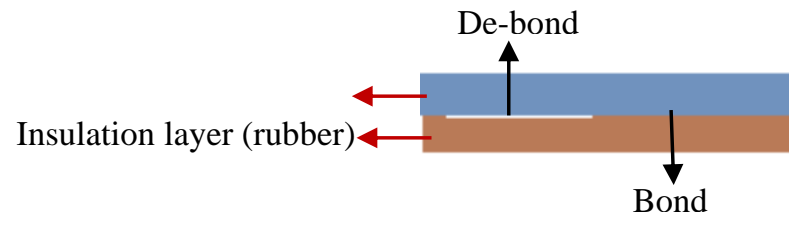

Fig. 5. Configuration of sample showing bond and debond regions

The reflection characteristics consisting of magnitude and phase for E-glass epoxy composite bonded to silicone rubber measured by Near-field reflection microwave NDE in contact mode are shown in Figure 6 and Figure 7 respectively. Once the experimental setup is calibrated, the reflection characteristics of the aluminium plate are measured for reference. The sample backed by an aluminum plate is inspected by an Open-ended rectangular waveguide probe in the frequency range of $8.2-12.4 \mathrm{GHz}$ (X-band). The reflection characteristics of a sample depending on the material dielectric properties, material thickness and its response to frequency. The sample configuration for the bond region is E-glass epoxy composite bonded to silicone rubber. For the debond region, the configuration is E-glass epoxy composite and silicone rubber separated by a thin layer of air-gap.

From the results, the bond and debond regions are distinguishable. The debond curve shifts to lower frequency as compared to the bond curve. It is observed from the reflection magnitude characteristics, the bond quality of the inspected structure is sensitive in the frequency range of 9.6$12.1 \mathrm{GHz}$ and $9.8-10.2 \mathrm{GHz}$ from the reflection phase characteristics respectively. During the inspection, the test frequency is swept and hence the technique is also called a Swept frequency reflection microwave NDE technique.

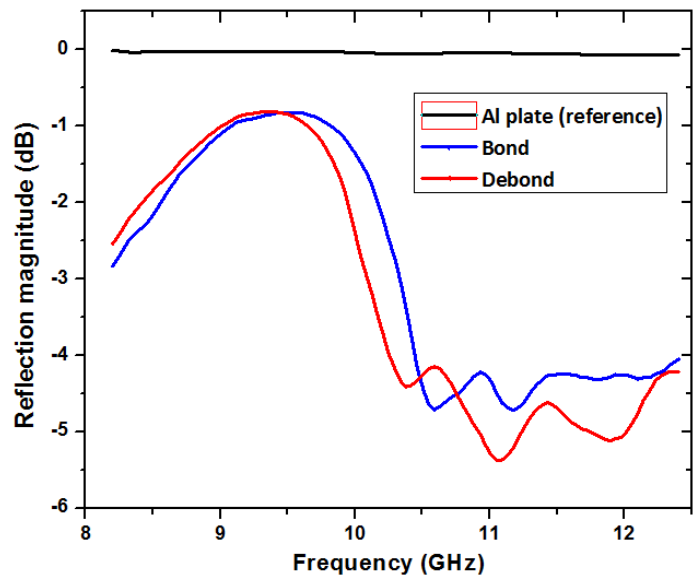

Fig. 6. Reflection magnitude of E-glass epoxy- silicone rubber at $\mathrm{X}$-band 


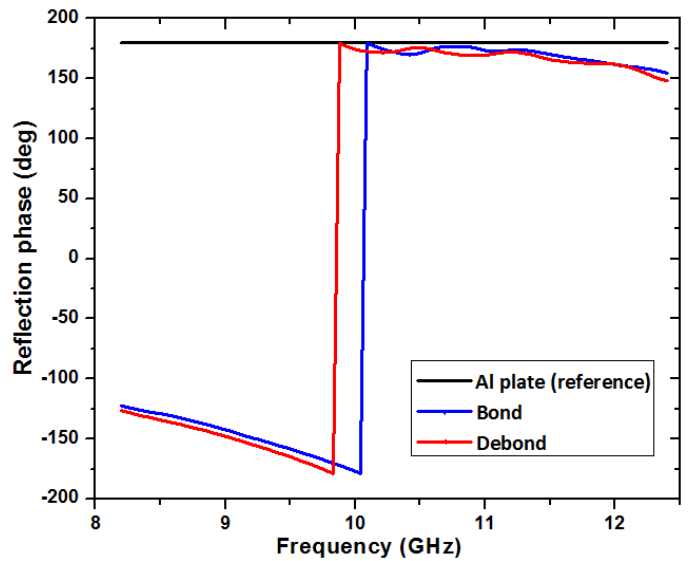

Fig. 7. Reflection phase of E-glass epoxy- silicone rubber at Xband

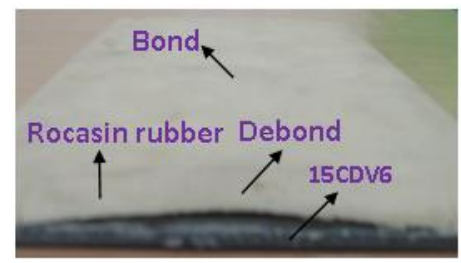

Fig. 8. 15CDV6 metal with rocasin rubber insulation showing bond and debond regions

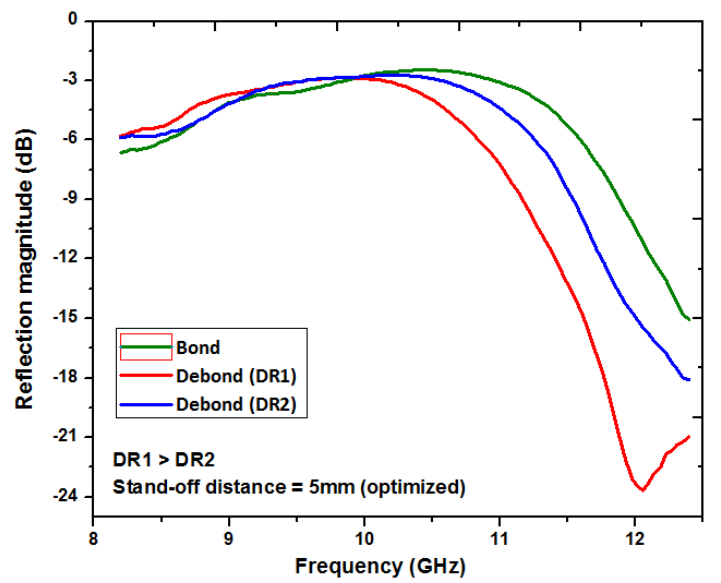

Fig. 9. Reflection magnitude of 15CDV6 metal- rocasin rubber at X-band

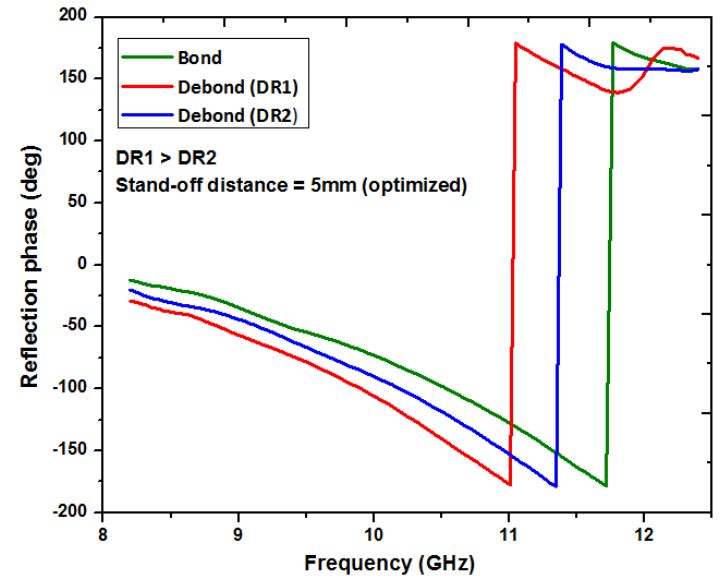

Fig. 10. Reflection phase of 15 CDV6 metal - rocasin rubber at Xband
Debond region 1 has a greater thickness than that of debond region 2 . The stand-off distance is varied manually from $0 \mathrm{~mm}$ (contact) to $7 \mathrm{~mm}$. For the given structure and test frequency, stand-off distance is optimized to detect the bond and debond regions. The optimized stand-off distance is $5 \mathrm{~mm}$ where the bond and de-bond regions are distinguishable. From the magnitude and reflection characteristics, the bond and debond regions are clearly distinguishable. It is observed from the results, the debond curve shifts to lower frequency as compared to the bond curve. Also, the position of the characteristic curve indicates the debond thickness. The greater the debond thickness, the larger is the shift in the characteristic curve. The bond quality of the inspected structure is sensitive in the frequency range of $10.3-12.4 \mathrm{GHz}$ from the reflection magnitude characteristics and $8.2-12 \mathrm{GHz}$ from the reflection phase characteristics respectively. Either magnitude or phase characteristics can be an indicative parameter of bond quality. Further, the position of the debond curve can be used to quantify the debond thickness.

The inspection of the composite pressure vessel (CPV) model shown in Figure 11, is carried out in the frequency range of $12.4-18 \mathrm{GHz}$ (Ku-band) by contact method. A customized fixture for holding the Open-ended rectangular waveguide probe is used for the inspection as shown in Figure 12.

The composite structure referred here consists of carbon epoxy composite with rocasin rubber as an insulation layer. The composite casing structure is fabricated by unidirectional carbon epoxy (CE) material having both helical and hoop filament winding methods. Because of the orientation of carbon epoxy material in the fabricated structure, the penetration of microwaves is limited. This makes carbon epoxy composite casing to have similar reflection characteristics as that of a conductor. In this paper, inspection is carried out on structure having carbon epoxy composite casing with rocasin rubber insulation.

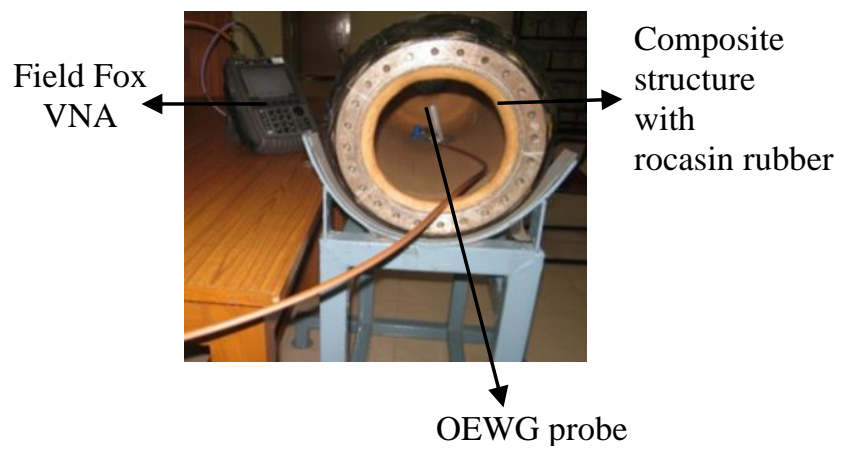

Fig. 11. Experimental setup for inspecting composite pressure vessel with rocasin rubber insulation by contact method

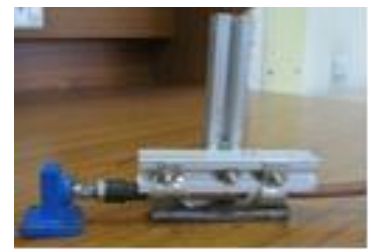

Fig.12. Customized fixture for holding OEWG probe 


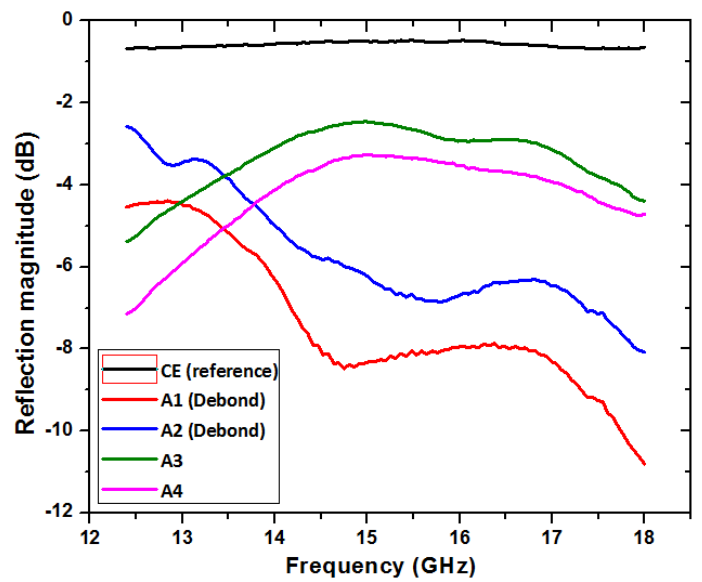

Fig. 13. Reflection magnitude of CPV at Ku-band

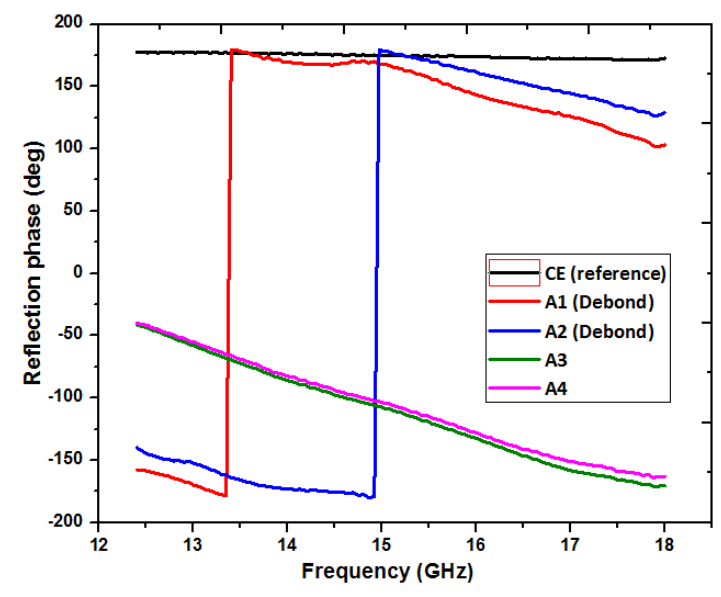

Fig. 14. Reflection phase of CPV at Ku-band

The magnitude and phase reflection characteristics of the composite structure (CPV) with rocasin rubber insulation are shown in Figures 13 and 14, respectively. Carbon epoxy (CE) composite acts as a conductor backup and is taken as a reference. Results pertaining to two non-defect (bond) regions and two defects (debond) regions are reported. Characteristic curves A3 and A4 correspond to bond regions respectively. Characteristic curves $\mathrm{A} 1$ and $\mathrm{A} 2$ correspond to debond regions respectively where debond thickness of A1 is greater than that of A2. From the results, the bond and debond regions are distinguishable. From the reflection phase results of debond characteristics, the position of the curve indicates the debond thickness.

The bond quality of the inspected CPV is sensitive in the frequency range of $14-18 \mathrm{GHz}$ from the reflection magnitude characteristics and $12-18 \mathrm{GHz}$ from the reflection phase characteristics respectively.

Swept frequency reflection microwave NDE technique is automated to provide microwave imaging of the inspected structure. Swept frequency reflection microwave NDE system consists of Keysight Vector network analyzer (VNA), XYZ scanner, coaxial cables, calibration kit, integration software, sample under test backed by conductor and open-ended rectangular waveguide probe. The integration software contains software for motion controlling of scanner and data acquisition. The microwave NDE system is shown in Figure 15. The microwave NDE system is developed for inspecting flat dielectric structures.

The bond quality of E-glass epoxy composite with silicone rubber and $15 \mathrm{CDV} 6$ metal with rocasin rubber is inspected by swept frequency reflection microwave NDE system respectively in the frequency range $8.2-18 \mathrm{GHz}$. The structures to be inspected are scanned by the microwave NDE system for the frequency of interest and reflection data consisting of magnitude and phase are collected for each scan location along with the scan position (X and $\mathrm{Y}$ ) respectively. Frequency sensitive to the bond and debond regions is identified from each of the reflection magnitude and reflection phase data respectively and the corresponding data is represented as a microwave $\mathrm{C}$-scan image.

Figure 16 shows the microwave image from the reflection magnitude data representing the bond quality of E-glass epoxy composite with silicone rubber at a frequency of 15.03GHz. The bond and debond regions are clearly distinguishable. For a given structure, the magnitude or phase response of bond and debond regions respectively to a particular frequency will determine the detection sensitivity of the inspection technique. The results of bond quality inspection by swept-frequency reflection microwave NDE technique are in agreement with the results of the thermography technique. Figure 17 shows the thermography image of bond quality of E-glass epoxy composite with silicone rubber.

Figure 18 shows the microwave image from the reflection phase data representing the bond quality of 15CDV6 metal with rocasin rubber at a frequency of $11.2 \mathrm{GHz}$. In this case, the two debond regions with different thickness is clearly noticeable in the microwave $\mathrm{C}$-scan image.

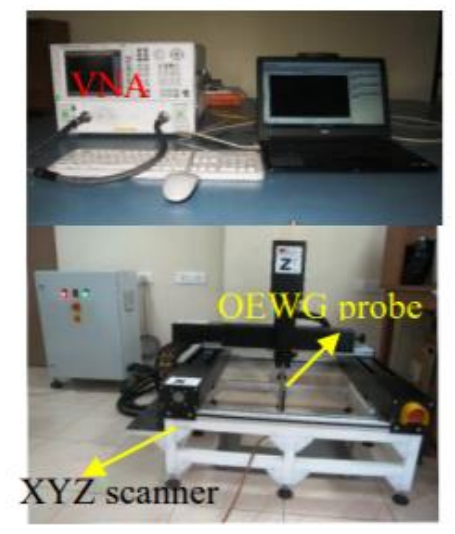

Fig. 15. Swept frequency reflection microwave NDE system

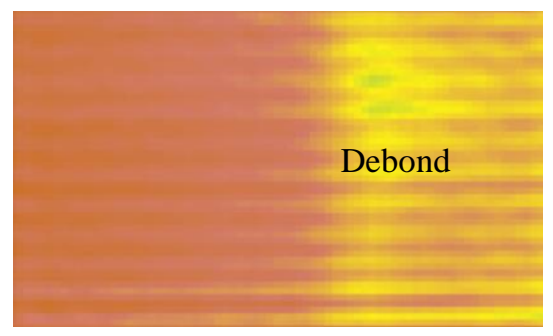

Fig.16. Microwave image representing bond quality of E-glass epoxy composite with silicone rubber at $15.03 \mathrm{GHz}$ (magnitude data) from Swept frequency reflection microwave NDE system 


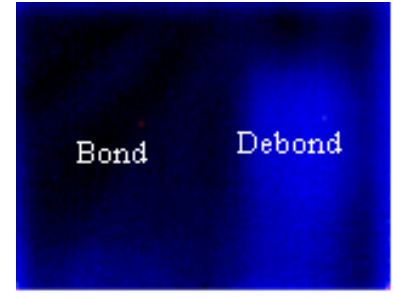

Fig. 17. Thermography image representing bond quality of E-glass epoxy composite with silicone rubber

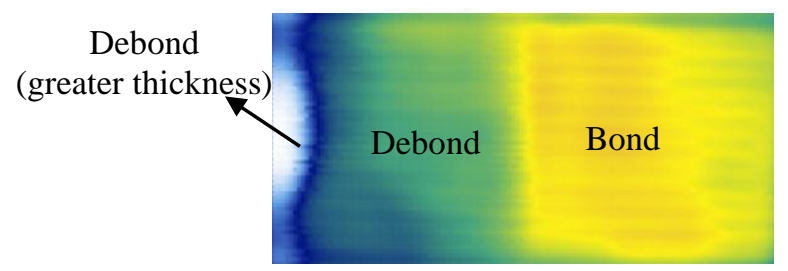

Fig. 18. Microwave image representing bond quality of $15 \mathrm{CDV} 6$ metal with rocasin rubber at $11.2 \mathrm{GHz}$ (phase data) from Swept frequency reflection microwave NDE system

\section{Conclusion}

Microwave nondestructive evaluation (MWNDE) is an emerging NDE technique for characterizing and inspecting dielectric composite structures. Microwave NDE is a useful tool for qualitative and quantitative analysis of bond quality inspection of structures consisting of composite or metal casing with an insulation layer. Microwave NDE provides single-sided and non-contact inspection over a range of testing frequencies. Swept frequency reflection microwave NDE is a complementary technique to evaluate dielectric structures.

\section{Acknowledgement}

The authors are thankful to the Director, Advanced Systems Laboratory (ASL) for providing an opportunity, support and encouragement to carry out the work.

\section{References}

[1] Evaluation, Non-destructive evaluation series volume 4, Kluwer Academic Publishers, 2000.

[2] N. Ida, Microwave NDT, Developments in Electromagnetic theory and applications, volume 10, Kluwer Academic Publishers, 1992.

[3] Asha Gokul, K. Srinivas, D. Radhika and J. Dhanasekaran, Application of Microwave NDE for Dielectric FRP Composites, International Conference on Composite Materials and Structures- ICCMS 2017.

\section{Biography of the author}

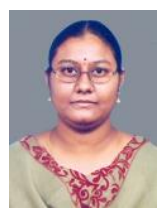

Asha Gokul received Bachelor of Engineering (B. E) degree in Electronics and Communication Engineering and Masters of Engineering (M. E) degree in Microwave and Radar Engineering from Osmania University, Hyderabad, India. She is currently working as Scientist in Advanced Systems Laboratory, DRDO, Hyderabad. Her areas of work include Electromagnetic design of radomes, Dielectric matrial characterization and Microwave NDE.

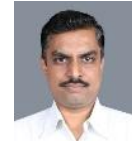

K. Srinivas received $\mathrm{PhD}$ degree in Physics from Osmania University, Hyderababd, India. $\mathrm{He}$ is currently working as Scientist in Advanced Systems Laboratory, DRDO, Hyderabad. His areas of work include NDE of Polymeric Composites and developing various NDE techniques. 